\title{
Capturing the oxidation of silicon carbide in rocky exoplanetary interiors
}

\author{
Kaustubh Hakim ${ }^{1,2}$, Wim van Westrenen ${ }^{2}$, and Carsten Dominik ${ }^{1}$ \\ 1 Anton Pannekoek Institute for Astronomy, University of Amsterdam, Science Park 904, 1098 XH Amsterdam, The Netherlands \\ e-mail: hakim.kaustubh@gmail.com \\ 2 Department of Earth Sciences, Vrije Universiteit, De Boelelaan 1085, 1081 HV Amsterdam, The Netherlands
}

Received 24 July 2018 / Accepted 24 September 2018

\begin{abstract}
Context. Theoretical models predict the condensation of silicon carbide around host stars with $\mathrm{C} / \mathrm{O}$ ratios higher than $0.65\left(\mathrm{cf} . \mathrm{C} / \mathrm{O}_{\text {Sun }}=\right.$ 0.54), in addition to its observations in meteorites, interstellar medium and protoplanetary disks. Consequently, the interiors of rocky exoplanets born from carbon-enriched refractory material are often assumed to contain large amounts of silicon carbide.

Aims. Here we aim to investigate the stability of silicon carbide in the interior of carbon-enriched rocky exoplanets and to derive the reaction leading to its transformation.

Methods. We performed a high-pressure high-temperature experiment to investigate the reaction between a silicon carbide layer and a layer representative of the bulk composition of a carbon-enriched rocky exoplanet.

Results. We report the reaction leading to oxidation of silicon carbide producing quartz, graphite, and molten iron silicide. Combined with previous studies, we show that in order to stabilize silicon carbide, carbon saturation is not sufficient, and a complete reduction of $\mathrm{Fe}^{2+}$ to $\mathrm{Fe}^{0}$ in a planetary mantle is required, suggesting that future spectroscopic detection of $\mathrm{Fe}^{2+}$ or $\mathrm{Fe}^{3+}$ on the surface of rocky exoplanets would imply the absence of silicon carbide in their interiors.
\end{abstract}

Key words. planets and satellites: terrestrial planets - planets and satellites: composition - planets and satellites: interiors planets and satellites: surfaces - methods: laboratory: molecular

\section{Introduction}

Silicon carbide grains have been observed in meteorites (Huss et al. 2003), in the interstellar medium (Min et al. 2007), and in protoplanetary disks (Fujiyoshi et al. 2015). Although the Earth is poor in carbon, carbon-rich carbonaceous chondrite meteorites, diamonds discovered in the ureilite parent body (Nabiei et al. 2018), and the proposed presence of graphite on Mercury's surface (Peplowski et al. 2016) suggest locally carbon-rich environments in the early solar system. Moreover, chemical simulations of protoplanetary disks around host stars with $\mathrm{C} / \mathrm{O}$ ratios higher than $0.65\left(\mathrm{cf} . \mathrm{C} / \mathrm{O}_{\text {Sun }}=0.54\right.$ ) result in the condensation of refractory minerals ranging from oxides, silicates, and metals to silicon carbide and graphite (Bond et al. 2010; Carter-Bond et al. 2012; Moriarty et al. 2014). Dynamical simulations show that these refractory minerals end up in the interiors of rocky exoplanets in different proportions with up to $47 \mathrm{wt} \%$ carbon (Carter-Bond et al. 2012). Because of the low density of the carbon-bearing minerals, silicon carbide and graphite are used to explain the low-density rocky exoplanets in the mass-radius diagram with insignificant gas envelopes (Seager et al. 2007; Madhusudhan et al. 2012).

Because of speculations of silicon carbide in exoplanetary interiors, physical properties of silicon carbide are being studied extensively at high pressures and temperatures (Wilson \& Militzer 2014; Nisr et al. 2017; Daviau \& Lee 2017; Miozzi et al. 2018). Significant amounts of silicon carbide in exoplanetary interiors would have a major impact on the thermal evolution and geodynamical processes on such exoplanets because its thermal conductivity is abnormally high (Nisr et al. 2017). There is no question that silicon carbide is highly refractory in nature because its extremely high melting temperatures facilitate its survival in protoplanetary disks once formed. However, the pressures in the interior of planets are orders of magnitude higher than those in protoplanetary disks, which strongly affects its stability.

Laboratory experiments suggest that $\mathrm{SiC}$ is not stable at high-pressure high-temperature conditions resembling those in carbon-enriched exoplanetary interiors (Hakim et al. 2018). Moissanite (naturally occurring $\mathrm{SiC}$ ), a rare mineral in the Earth, is known to be unstable in the carbon-poor conditions dominating Earth's mantle and crust, and its formation in Earth is attributed to extremely reducing local conditions (Schmidt et al. 2014; Golubkova et al. 2016). Experiments in the $\mathrm{Fe}-\mathrm{Mg}-\mathrm{Si}-\mathrm{C}-\mathrm{O}(\mathrm{FMS}+\mathrm{CO})$ system show that silicon carbide is stable only at extremely low oxygen fugacities of about $6 \log$ units below the iron-wüstite (IW) buffer $\left(\log f_{\mathrm{O}_{2}}=\mathrm{IW}-6\right)$ (Takahashi et al. 2013). At oxygen fugacities above IW - 6, silicon carbide becomes oxidized, but it is not clear which reaction drives the instability of silicon carbide in a carbon-enriched exoplanetary interior. In this study, we performed an experiment at $1 \mathrm{GPa}$ and $1823 \mathrm{~K}$ by juxtaposing an $\mathrm{SiC}$ layer and a bulk composition representative of a small carbon-enriched rocky exoplanet (Hakim et al. 2018; see Table 1). 
Table 1. Bulk composition of a carbon-enriched planetesimal based on protoplanetary disk evolution modeling around high $\mathrm{C} / \mathrm{O}$ stars from Moriarty et al. (2014).

\begin{tabular}{lrlr}
\hline \hline Element & mol\% & Material & wt\% \\
\hline $\mathrm{Si}$ & 11.4 & $\mathrm{SiO}_{2}$ & 30.1 \\
$\mathrm{Mg}$ & 11.4 & $\mathrm{MgO}$ & 20.2 \\
$\mathrm{O}$ & 45.8 & $\mathrm{FeO}^{a}$ & 27.3 \\
$\mathrm{Fe}$ & 11.4 & $\mathrm{Fe}$ & 2.2 \\
$\mathrm{~S}$ & 1.9 & $\mathrm{FeS}$ & 7.0 \\
$\mathrm{Al}$ & 1.4 & $\mathrm{Al}_{2} \mathrm{O}_{3}$ & 3.1 \\
$\mathrm{Ca}$ & 0.7 & $\mathrm{CaO}^{a}$ & 1.7 \\
$\mathrm{C}$ & 16.0 & $\mathrm{C}$ & 8.4 \\
\hline
\end{tabular}

Notes. (a) $\mathrm{CaO}$ and $\mathrm{FeO}$ are obtained from $\mathrm{CaCO}_{3}$ and $\mathrm{Fe}_{2} \mathrm{O}_{3}$ after decarbonation. The starting composition reflects the composition of the powder after the decarbonation.

\section{Experimental and analytical method}

\subsection{Starting materials}

We prepared a mixture of eight major elements (Fe, O, Si, Mg, $\mathrm{Al}, \mathrm{Ca}, \mathrm{S}$, and $\mathrm{C}$ ) representative of the bulk composition of a carbon-enriched exoplanet. The proportions of elements in these mixtures are based on the sequential condensation modeling of the protoplanetary disk of HD19994 at 1 astronomical unit (AU) and 0.15 Myr from the study by Moriarty et al. (2014; see Table 1). To prepare the chemical mixtures, the starting materials were mixed in proportions shown in Table 1. In the first step, $\mathrm{SiO}_{2}\left(99.9 \% \mathrm{SiO}_{2}\right.$ powder from Alfa-Aesar), $\mathrm{MgO}\left(99.95 \% \mathrm{MgO}\right.$ powder from Alfa-Aesar), $\mathrm{Al}_{2} \mathrm{O}_{3}(99.95 \%$ min alpha $\mathrm{Al}_{2} \mathrm{O}_{3}$ powder from Alfa-Aesar), $\mathrm{CaCO}_{3}$ (99.95$100.05 \%$ ACS chelometric standard $\mathrm{CaCO}_{3}$ powder from AlfaAesar), and $\mathrm{Fe}_{2} \mathrm{O}_{3}\left(99.9 \% \mathrm{Fe}_{2} \mathrm{O}_{3}\right.$ powder from Alfa-Aesar) were homogenized with an agate mortar under ethanol. The oxidecarbonate mixture was decarbonated in a box furnace by gradually increasing the temperature from $873 \mathrm{~K}$ to $1273 \mathrm{~K}$ in six hours. The decarbonated mixture, placed in a Pt-crucible, was first subjected to $1873 \mathrm{~K}$ in a box furnace for 30 minutes and then quenched to room temperature by immersing the bottom of the Pt-crucible in water, leading to the formation of glass. The glass was ground to a homogeneous powder using an agate mortar under ethanol. Fe $(99.95 \%$ Fe powder, spherical, $<10$ microns from Alfa-Aesar) and $\mathrm{FeS}(99.9 \% \mathrm{FeS}$ powder from Alfa-Aesar) were then added to the glass powder. In the carbon-enriched case, C $(99.9995 \%$ Ultra F purity graphite powder from AlfaAesar) was also added to the glass powder. The final mixture was again homogeneously ground with an agate mortar and stored in an oven at $383 \mathrm{~K}$ until use. SiC ( -400 mesh particle size, $\geq 97.5 \%$ $\mathrm{SiC}$ from Alfa-Aesar) was also ground with an agate mortar and stored separately.

\subsection{High-pressure high-temperature experiments}

The experiment was conducted in an end-loaded piston-cylinder apparatus at a pressure of $1 \mathrm{GPa}$ and temperature of $1823 \mathrm{~K}$ in a $12.7 \mathrm{~mm}$ (half-inch) sample assembly. Carbon-enriched composition powder was inserted in a $1.6 \mathrm{~mm}$ wide graphite capsule, filling the capsule approximately $60 \%$ by volume. Silicon carbide powder was inserted on top of the carbon-enriched planetary bulk composition, filling the remaining $40 \%$ by volume of the capsule. The capsule was then sealed with a stepped graphite lid. This graphite capsule was put into a $2 \mathrm{~mm}$ wide $\mathrm{Pt}$ capsule that was sealed and arc-welded on both ends using a Lampert PUK 3 welder. The Pt capsule was placed in a $\mathrm{MgO}$ rod sealed with $\mathrm{MgO}$ cement to hold the Pt capsule in place. The $\mathrm{MgO}$ rod was introduced in a graphite furnace, thermally insulated by surrounding it with an inner pyrex sleeve and an outer talc sleeve. A four-bore $\mathrm{Al}_{2} \mathrm{O}_{3}$ rod through which thermocouple wires were threaded was placed on the top of $\mathrm{MgO}$ rod. Pressure calibration of the assembly was performed by bracketing the albite to jadeite plus quartz and fayalite to ferrosilite plus quartz transitions (van Kan Parker et al. 2011). The resulting pressure correction of $3 \%$ is consistent with literature data (McDade et al. 2002). A hardened silver steel plug with a pyrophillite ring and a hole for thermocouple were placed on top of the talc-pyrex assembly. $\mathrm{A} \mathrm{W}_{97} \mathrm{Re}_{3} / \mathrm{W} 75 \mathrm{Re}_{25}$ (type D) thermocouple was placed in the thermocouple hole directly above the Pt capsule. The distance of $1-3.5 \mathrm{~mm}$ between the thermocouple tip and the sample produced a temperature difference of $\sim 10 \mathrm{~K}$ (Watson et al. 2002). To reduce the porosity of the graphite capsule, the sample assembly was sintered at $1073 \mathrm{~K}$ and $1 \mathrm{GPa}$ for $1 \mathrm{~h}$ before further heating and pressurization. During heating to run temperature, the pressure was increased continuously using the hot-piston-in technique. The temperature was increased at a rate of $100 \mathrm{~K} \mathrm{~min}^{-1}$. The experiment was run for the duration of $3.5 \mathrm{~h}$ and was subsequently quenched to $<500 \mathrm{~K}$ within $\sim 15 \mathrm{~s}$ by switching off the electric power to the heater.

\subsection{Analytical procedure}

The recovered samples were mounted in one-inch diameter mounts using petropoxy resin, cut longitudinally, polished with grit-paper and fine-polished down to a $1 / 4 \mu \mathrm{m}$ finish. The polished samples were carbon-coated to ensure electrical conductivity of the surface during electron probe micro-analysis (EPMA). Major element contents of the experimental charges were determined using wavelength dispersive spectroscopy on the fivespectrometer JEOL JXA-8530F Hyperprobe Field Emission Electron probe micro-analyzer at the Netherlands National Geological Facility, Utrecht University. For $\mathrm{Fe}-\mathrm{C}$ and $\mathrm{Fe}-\mathrm{Si}$ alloys, samples were coated with aluminum instead of carbon, and analyses were performed using a JEOL JXA 8530F Hyperprobe at Rice University, Houston following the analytical protocol (Dasgupta \& Walker 2008). We used a series of silicate, oxide, and metal standards and conditions of $15 \mathrm{nA}$ beam current and $15 \mathrm{kV}$ accelerating voltage. Analyses were made with a defocused beam to obtain the compositions of the metal $(2-10 \mu \mathrm{m}$ diameter) and silicate (5-20 $\mu \mathrm{m}$ diameter) phases. Standards for the quantitative analysis of $\mathrm{Mg}, \mathrm{Fe}, \mathrm{Si}, \mathrm{Al}$, and $\mathrm{Ca}$ in silicate minerals were forsterite, hematite, forsterite, corundum, and diopside, respectively. Standards used for measuring Fe, Si, C, S, and $\mathrm{O}$ were $\mathrm{Fe}$-metal, $\mathrm{Si}$-metal, experimentally synthesized $\mathrm{Fe}_{3} \mathrm{C}$, natural troilite, and magnetite, respectively. Counting times were $30 \mathrm{~s}$ for $\mathrm{Fe}$ (hematite and $\mathrm{Fe}$-metal), $\mathrm{Si}, \mathrm{C}, \mathrm{O}, \mathrm{Mg}$, and $\mathrm{Al}$, and $20 \mathrm{~s}$ for $\mathrm{Ca}$ and S. Data reduction was performed using the $\Phi(\mathrm{rZ})$ correction (Armstrong 1995). The instrument calibration was deemed successful when the composition of secondary standards was reproduced within the error margins defined by the counting statistics.

\section{Results and discussion}

The backscattered electron image (Fig. 1a) of the experimental run product shows a clear reaction zone between the $\mathrm{SiC}$ layer (top) and the silicate-rich layer representing a carbon-enriched 
Table 2. Composition of silicate phases.

\begin{tabular}{lrrrrrrrr}
\hline \hline Phase & $n$ & $\mathrm{SiO}_{2}$ & $\mathrm{MgO}$ & $\mathrm{FeO}$ & $\mathrm{Al}_{2} \mathrm{O}_{3}$ & $\mathrm{CaO}$ & $S$ & $\mathrm{Sum}$ \\
\hline Quartz specks & 4 & $99.64(0.49)$ & $0.20(0.17)$ & $1.06(0.17)$ & $0.15(0.06)$ & $0.05(0.03)$ & $0.07(0.05)$ & $101.15(0.23)$ \\
Silicate melt specks & 9 & $62.86(0.08)$ & $19.92(0.15)$ & $5.02(0.05)$ & $6.44(0.05)$ & $4.46(0.03)$ & $0.13(0.01)$ & $98.84(0.08)$ \\
Silicate melt pool & 20 & $61.81(0.21)$ & $20.60(0.44)$ & $5.01(0.15)$ & $6.77(0.08)$ & $4.47(0.24)$ & $0.14(0.00)$ & $98.79(0.23)$ \\
Orthopyroxene & 10 & $58.31(0.22)$ & $36.38(0.11)$ & $4.12(0.15)$ & $0.56(0.02)$ & $0.37(0.01)$ & $<\mathrm{DL}$ & $99.73(0.12)$ \\
Olivine (bottom) & 12 & $41.47(0.29)$ & $52.95(0.26)$ & $5.48(0.24)$ & $0.12(0.05)$ & $0.11(0.02)$ & $<\mathrm{DL}$ & $100.13(0.19)$ \\
Silicate melt (bottom) & 8 & $53.37(0.42)$ & $26.45(1.63)$ & $6.16(0.52)$ & $8.21(0.55)$ & $4.28(0.88)$ & $0.10(0.03)$ & $98.58(0.83)$ \\
\hline
\end{tabular}

Notes. All compositions are in wt $\%$ with $1 \sigma$ error given in parantheses. $n$ is the number of analytical points. $<$ DL implies the measurements were below the detection limit. Pt contamination in all metallic phases was below the detection limit of approximately $0.07 \mathrm{wt} \%$. At these levels the activity of iron in these metal phases is unaffected (e.g., Steenstra et al. 2018a, and references therein).

Table 3. Composition of metallic phase.

\begin{tabular}{|c|c|c|c|c|c|c|c|}
\hline Phase & $n$ & $\mathrm{Fe}$ & $\mathrm{Si}$ & $\mathrm{C}$ & $\mathrm{S}$ & $\mathrm{O}$ & Sum \\
\hline $\mathrm{SiC}$ specks & 7 & $0.38(0.03)$ & $73.30(0.18)$ & $26.32(0.09)^{a}$ & $<\mathrm{DL}$ & - & 100 \\
\hline $\mathrm{Fe}-\mathrm{Si}$ specks & 7 & $78.40(0.09)$ & $19.83(0.29)$ & $0.94(0.18)^{b}$ & $0.12(0.01)$ & - & $99.29(0.18)$ \\
\hline Fe-Si bleb & 10 & $79.66(0.10)$ & $18.82(0.24)$ & $1.01(0.15)^{b}$ & $0.15(0.01)$ & - & $99.64(0.15)$ \\
\hline $\mathrm{Fe}-\mathrm{C}$ bleb & 11 & $93.75(0.41)$ & $<\mathrm{DL}$ & $4.33(0.10)$ & $0.79(0.14)$ & $0.64(0.07)$ & $98.87(0.23)$ \\
\hline $\mathrm{Fe}-\mathrm{S}$ rim & 8 & $65.90(2.01)$ & $<\mathrm{DL}$ & $1.66(0.79)$ & $31.22(2.03)$ & $0.74(0.59)$ & $98.78(1.51)$ \\
\hline
\end{tabular}

Notes. All compositions are in wt $\%$ with $1 \sigma$ error given in parantheses. $n$ is the number of analytical points. $<$ DL implies the measurements were below the detection limit. - implies lack of measurements. ${ }^{(a)}$ Calculated by subtracting the total from $100 .{ }^{(b)} \mathrm{C}$-abundance calculated using a model for C-solubility in Fe-Si from Steenstra et al. (2018b).

rocky exoplanet (bottom). Tables 2 and 3 give the compositions of the phases analyzed using wavelength-dispersive spectroscopy, whereas graphite was identified using energydispersive spectroscopy. The reaction zone contains grains of $\mathrm{C}$ (graphite) and $\mathrm{SiO}_{2}$ (quartz), and molten $\mathrm{Fe}-\mathrm{Si}$ alloy (iron silicide; Fig. 1b). SiC grains become oxidized in the reaction zone and no $\mathrm{SiC}$ is present in or below the reaction zone. Although most of $\mathrm{Fe}-\mathrm{Si}$ alloy moves to the silicate-rich layer, a small portion of it moves up through the silicon carbide layer. The silicate melt pool present below the reaction zone is enriched in $\mathrm{SiO}_{2}$ compared to the silicate melt at the bottom of the capsule because of the progressive dissolution of quartz formed in the reaction zone.

Previous experiments by Hakim et al. (2018) have shown that the equilibrium phases for the bottom silicate-rich layer representing a carbon-enriched rocky exoplanet comprise graphite, olivine, silicate melt, and a S-rich $\mathrm{Fe}-\mathrm{C}-\mathrm{S}$ alloy. In the presence of $\mathrm{SiC}$ grains, the silicate phases in the silicate-rich layer become richer in $\mathrm{MgO}$ and poorer in $\mathrm{FeO}$ compared to compositions formed in the absence of the initial SiC layer. Quartz produced during the process reacts with olivine to produce orthopyroxene. The $\mathrm{FeO}$ contents of olivine and silicate melt in the previous study (Hakim et al. 2018) were $34.2 \pm 1.1 \mathrm{wt} \%$ and $21.3 \pm 0.1 \mathrm{wt} \%$, respectively, whereas we find the $\mathrm{FeO}$ content of olivine, orthopyroxene, and silicate melt to be 4-6 wt $\%$. There is about five times less $\mathrm{FeO}$ in the silicate-rich layer of our experiment than its equilibrium state in the absence of the $\mathrm{SiC}$ layer. Moreover, the formation of $\mathrm{Fe}-\mathrm{Si}$ and $\mathrm{S}$-poor $\mathrm{Fe}-\mathrm{C}$ alloy melts implies that most of the $\mathrm{Fe}^{2+}$ initially bonded to oxygen is reduced to $\mathrm{Fe}^{0}$ in this reaction. Since the reaction products observed in the reaction zone are quartz, iron silicide melt, and graphite, we report the following reaction consuming silicon carbide:

$3 \mathrm{SiC}(\mathrm{s})+2 \mathrm{Fe}-\mathrm{O}(\mathrm{l}) \rightarrow \mathrm{SiO}_{2}(\mathrm{~s})+2 \mathrm{Fe}-\mathrm{Si}(\mathrm{l})+3 \mathrm{C}(\mathrm{s})$.
In the equation, $\mathrm{Fe}-\mathrm{O}$ (1) denotes $\mathrm{Fe}^{2+}$ bonded to oxygen in silicate melt, and $\mathrm{Fe}-\mathrm{Si}$ (l) denotes $\mathrm{Fe}^{0}$ bonded to $\mathrm{Si}^{0}$ in metallic liquid. An important consequence of the reduction of $\mathrm{Fe}^{2+}$ is that the molar $\mathrm{Mg} /(\mathrm{Mg}+\mathrm{Fe})$ of olivine and other silicates $\left(X_{\mathrm{Mg}}\right)$ are very high because of the lack of $\mathrm{FeO}$. In our experiment we find $X_{\mathrm{Mg}} \sim 0.95$ for olivine and orthopyroxene and $X_{\mathrm{Mg}} \sim 0.88$ for the silicate melt, which are significantly higher than the equilibrium state (Hakim et al. 2018), $X_{\mathrm{Mg}} \sim 0.77$ for olivine and $X_{\mathrm{Mg}} \sim 0.47$ for the silicate melt. Experiments and theoretical modeling have shown that $\mathrm{SiC}$ and $\mathrm{Fe}-\mathrm{Si}$ alloy can be equilibrated with olivine only when its $X_{\mathrm{Mg}}>0.99$ (Schmidt et al. 2014; Golubkova et al. 2016). Experiments in carbon-saturated FMS+CO system also find $\mathrm{SiC}$ to be stable only when olivines have a very high $X_{\mathrm{Mg}}$ of 0.992 (Takahashi et al. 2013). We conclude that almost all $\mathrm{Fe}^{2+}$ should be in its reduced state, $\mathrm{Fe}^{0}$, to stabilize $\mathrm{SiC}$ even in a carbon-enriched rocky exoplanetary interior.

Since the reaction is still in progress, the conditions at the top of the silicate-rich layer are more reducing than at its bottom. Therefore, Fe-Si alloy is only present within the original $\mathrm{SiC}$ layer and at the top of the silicate-rich layer, whereas the remainder of the silicate layer contains $\mathrm{Fe} \pm \mathrm{C} \pm \mathrm{S}$ alloys only. One $\mathrm{Fe}-\mathrm{C}$ bleb at the bottom of the capsule is surrounded by a $\mathrm{Fe}-\mathrm{S}$ alloy showing liquid metal immiscibility because of the lowered local $\mathrm{S} / \mathrm{Fe}$ ratio, as shown in the previous study by Hakim et al. (2018; Fig. 1c). Similarly, orthopyroxene, which is relatively oxygenpoorer than olivine, is found in most of the silicate-rich layer except at the bottom where olivine is present. We do not find any $\mathrm{SiC}$ below the reaction zone, which also contains a large $\mathrm{Fe}-\mathrm{Si}$ bleb. This suggests that $\mathrm{SiC}$ forms at even more reducing conditions than needed for the formation of the Fe-Si alloy, which is in contrast to previous modeling studies in the context of moissanite stability in the Earth's mantle, which suggested that $\mathrm{SiC}$ and $\mathrm{Fe}-\mathrm{Si}$ alloy form together at the same oxygen fugacities (Schmidt et al. 2014; Golubkova et al. 2016). 

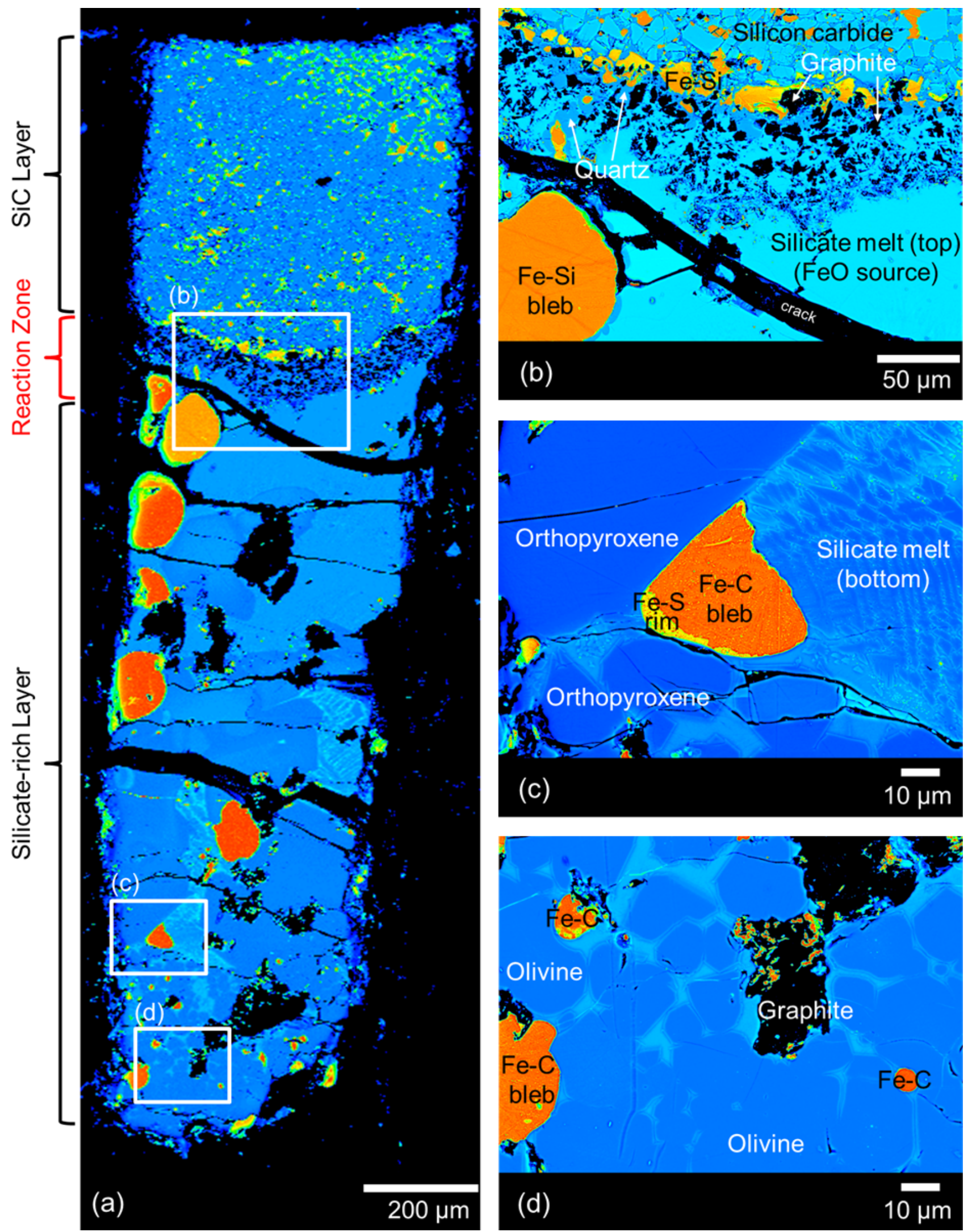

Fig. 1. False-color backscattered electron images of the run product at $1 \mathrm{GPa}$ and $1823 \mathrm{~K}$. Panel a: cross-section of the whole capsule is shown. The reaction zone lies between the silicon carbide layer and the silicate-rich layer representing a carbon-enriched rocky exoplanet. Panel $b$ : the reactants ( $\mathrm{SiC}$ and $\mathrm{FeO}$ from silicate melt) and the products (quartz, graphite, and $\mathrm{Fe}-\mathrm{Si}$ alloy) are clearly visible. Panel $c$ : one of the $\mathrm{Fe}-\mathrm{C}$ blebs has a $\mathrm{Fe}-\mathrm{S}$ rim, surrounded by orthopyroxene crystals and silicate melt. Panel $d$ : at the bottom of the capsule, olivine crystals are present instead of orthopyroxene. 
The backscattered electron image (Fig. 1a) of the experimental run product shows a clear reaction zone between the $\mathrm{SiC}$ layer (top) and the silicate-rich layer representing a carbonenriched rocky exoplanet (bottom). Tables 2 and 3 give the compositions of the phases analyzed using wavelength-dispersive spectroscopy, whereas graphite was identified using energydispersive spectroscopy. The reaction zone contains grains of $\mathrm{C}$ (graphite) and $\mathrm{SiO}_{2}$ (quartz), and molten $\mathrm{Fe}-\mathrm{Si}$ alloy (iron silicide; Fig. 1b). SiC grains become oxidized in the reaction zone and no $\mathrm{SiC}$ is present in or below the reaction zone. Although most of $\mathrm{Fe}-\mathrm{Si}$ alloy moves to the silicate-rich layer, a small portion of it moves up through the silicon carbide layer. The silicate melt pool present below the reaction zone is enriched in $\mathrm{SiO}_{2}$ compared to the silicate melt at the bottom of the capsule because of the progressive dissolution of quartz formed in the reaction zone.

Our experimental conditions are applicable to the magma ocean stage of Ceres- to Pluto-size exoplanets and planetesimals. Larger rocky exoplanets form from the collision and accretion of such planetesimals. Since silicon carbide is not stable in exoplanetary building blocks and based on the rapid pace of the reaction in our experiment, $\mathrm{SiC}$ is expected to completely disappear before the formation of larger exoplanets. There may still be cases where an exoplanetary interior is reducing, for example, through a bombardment of SiC-rich meteorites onto the protoplanet. In such a case, the core of the exoplanet will become larger because $\mathrm{FeO}$ in the mantle is reduced to $\mathrm{Fe}$ and formation of $\mathrm{Fe}-\mathrm{Si}$ alloy, which will move to the core. This will enrich the core with $\mathrm{Si}$, and the mantle will be deficient in $\mathrm{FeO}$ in this case.

In order for $\mathrm{SiC}$ to become a dominant mineral in a rocky exoplanet, our experiment indicates that the conditions should be so reducing that such a planet would already contain a $\mathrm{Fe}-$ $\mathrm{Si}$ alloy core, contrary to the assumption of a pure $\mathrm{Fe}$ core with a SiC-C mantle in previous studies (Madhusudhan et al. 2012; Nisr et al. 2017). Our results show that $\mathrm{SiC}$ is unstable until the conditions are extremely reducing, allowing only for traces of $\mathrm{Fe}^{2+}$, and previous studies (Takahashi et al. 2013; Hakim et al. 2018) show that graphite/diamond is the dominant carbon-bearing mineral. Hence, care should be taken when assuming $\mathrm{SiC}$ in the interior modeling of a carbon-enriched rocky exoplanet. Assuming no effects of atmosphere or water on the composition of the surface of a rocky exoplanet, if $\mathrm{Fe}^{2+}$ or even more oxidizing $\mathrm{Fe}^{3+}$ is present in the mantle, it should also be present on the surface. Since the presence of $\mathrm{Fe}^{2+}$ or $\mathrm{Fe}^{3+}$ implies the absence of $\mathrm{SiC}$, future spectroscopic detections of $\mathrm{Fe}^{2+}$ or even more oxidizing $\mathrm{Fe}^{3+}$ on the surface of a rocky exoplanet would mean that its interior is devoid of $\mathrm{SiC}$. The conversion of $\mathrm{SiC}$ into graphite as well as the presence of graphite in carbon-saturated but not extremely reduced rocky exoplanets would have important consequences for the surface composition and therefore for the habitability of such planets.

Acknowledgements. This work is part of the Planetary and Exoplanetary Science Network (PEPSci), funded by the Netherlands Organization for Scientific Research (NWO, Project no. 648.001.005). We are grateful to Sergei Matveev and Tilly Bouten from Utrecht University for their technical assistance during EPMA measurements at Utrecht University. We thank Damanveer Grewal and Rajdeep Dasgupta for performing and facilitating analyses of metals in the EPMA Laboratory at Rice University.

\section{References}

Armstrong, J. T. 1995, Microbeam Anal., 4, 177

Bond, J. C., O'Brien, D. P., \& Lauretta, D. S. 2010, ApJ, 715, 1050

Carter-Bond, J. C., O'Brien, D. P., \& Raymond, S. N. 2012, ApJ, 760, 44 Dasgupta, R., \& Walker, D. 2008, Geochim. Cosmochim. Acta, 72, 4627 Daviau, K., \& Lee, K. K. M. 2017, Phys. Rev. B, 96, 174102

Fujiyoshi, T., Wright, C. M., \& Moore, T. J. T. 2015, MNRAS, 451, 3371

Golubkova, A., Schmidt, M. W., \& Connolly, J. A. D. 2016, Contrib. Mineral. Petrol., 171, 41

Hakim, K., Spaargaren, R., Grewal, D. S., et al. 2018, Astrobiology, submitted, [arXiv:1807.02064]

Huss, G. R., Meshik, A. P., Smith, J. B., \& Hohenberg, C. M. 2003, Geochim. Cosmochim. Acta, 67, 4823

Madhusudhan, N., Lee, K. K. M., \& Mousis, O. 2012, ApJ, 759, L40

McDade, P., Wood, B. J., Van Westrenen, W., et al. 2002, Miner. Mag., 66, 1021

Min, M., Waters, L. B. F. M., de Koter, A., et al. 2007, A\&A, 462, 667

Miozzi, F., Morard, G., Antonangeli, D., et al. 2018, J. Geophys. Res. (Planets), 123

Moriarty, J., Madhusudhan, N., \& Fischer, D. 2014, ApJ, 787, 81

Nabiei, F., Badro, J., Dennenwaldt, T., et al. 2018, Nat. Commun., 9, 1327

Nisr, C., Meng, Y., MacDowell, A. A., et al. 2017, J. Geophys. Res. (Planets), 122,124

Peplowski, P. N., Klima, R. L., Lawrence, D. J., et al. 2016, Nat. Geosci., 9, 273 Schmidt, M. W., Gao, C., Golubkova, A., Rohrbach, A., \& Connolly, J. A. D. 2014, Prog. Earth Planet. Sci., 1, 27

Seager, S., Kuchner, M., Hier-Majumder, C. A., \& Militzer, B. 2007, ApJ, 669, 1279

Steenstra, E. S., Seegers, A. X., Eising, J., et al. 2018a, Geochim. Cosmochim. Acta, 231, 130

Steenstra, E. S., Seegers, A. X., Putter, R., et al. 2018b, Chem. Geol., submitted Takahashi, S., Ohtani, E., Terasaki, H., et al. 2013, Phys. Chem. Miner., 40, 647 van Kan Parker, M., Mason, P. R. D., \& van Westrenen, W. 2011, Cosmochim. Acta, 75, 4179

Watson, E. B., Wark, D. A., Price, J. D., \& Van Orman, J. A. 2002, Contrib. Mineral. Petrol., 142, 640

Wilson, H. F., \& Militzer, B. 2014, ApJ, 793, 34 